\title{
Research on Remote Monitoring Technologies of the Outdoor Advertising Screen
}

\author{
Jiang Jian ${ }^{1, a}$, Chen Zhang ${ }^{2, b}$, Ren Jie $^{3, c}$ \\ ${ }^{1}$ School of Mechanics, Nanjing University of Science and Technology Nanjing, China \\ ${ }^{2}$ School of Mechanics, Nanjing University of Science and Technology Nanjing, China \\ ${ }^{3}$ School of Mechanics, Nanjing University of Science and Technology Nanjing, China \\ a83905118@163.com, b460750242@qq.com, 'littleprecious@163.com
}

Key Words: outdoor advertising screen, remote monitor and control, C/S model, the Ethernet Abstract Based on the lack of monitoring methods and strength of the outdoor advertising screen remote monitoring technology, the overall framework of the remote monitoring system based on the $\mathrm{C} / \mathrm{S}$ mode is constructed and the overall design of the monitoring unit and the monitoring center of the advertisement screen are completed. Those are communicated by the Ethernet and the GSM. The experiment shows that the research result of the remote monitoring technology of the outdoor advertising screen has the characteristics of advanced technology, strong practicability and so on. The system function is comprehensive, stable operation, and can be popularized and used.

\section{Introduction}

With the tide of information and digitalization, the outdoor advertising industry in our country has reached a considerable size. In order to promote the city' image, the urban smart and the digital construction, the government departments of many large cities in China are required to set up outdoor dynamic LCD screens in public places such as bus stations, which has made further efforts to increase the demand of outdoor advertising screens [1]. However, the traditional outdoor advertising screen is more and more unable to meet the demand of the city's intelligent development. Effective monitoring of outdoor advertising screen is the premise to ensure its normal operation and effective management [2]. Therefore, this topic has made intensive study on outdoor advertising screen remote monitoring technology, and has solved the shortcomings of traditional outdoor advertising screen's lacking of monitoring means and strength.

\section{The overall design of the remote monitoring and control system of the advertisement screen}

This system is designed based on C/S model, composed of two parts: the advertisement screen's monitoring unit and center, as is shown in the Fig 2.1. The monitoring unit of the advertisement screen is responsible for monitoring a variety of status information and controlling the operation of the advertisement screen. The monitor circuit board is the core control part of the advertisement screen monitor unit, connecting with the backlight lamp board, the light box, the GPS antenna, the GSM module, a variety of sensors, the audio power amplifier, transformers, fans and other devices to achieve the monitoring function of the advertising screen [3]. The advertisement screen monitoring unit controls the PC switch machine and the reset through the I/O line, communicating with the screen's PC through RS232, receiving the audio signal from the screen's PC through the audio. The monitoring center is responsible for state monitoring, image display, image storage, remote controlling and other functions. 


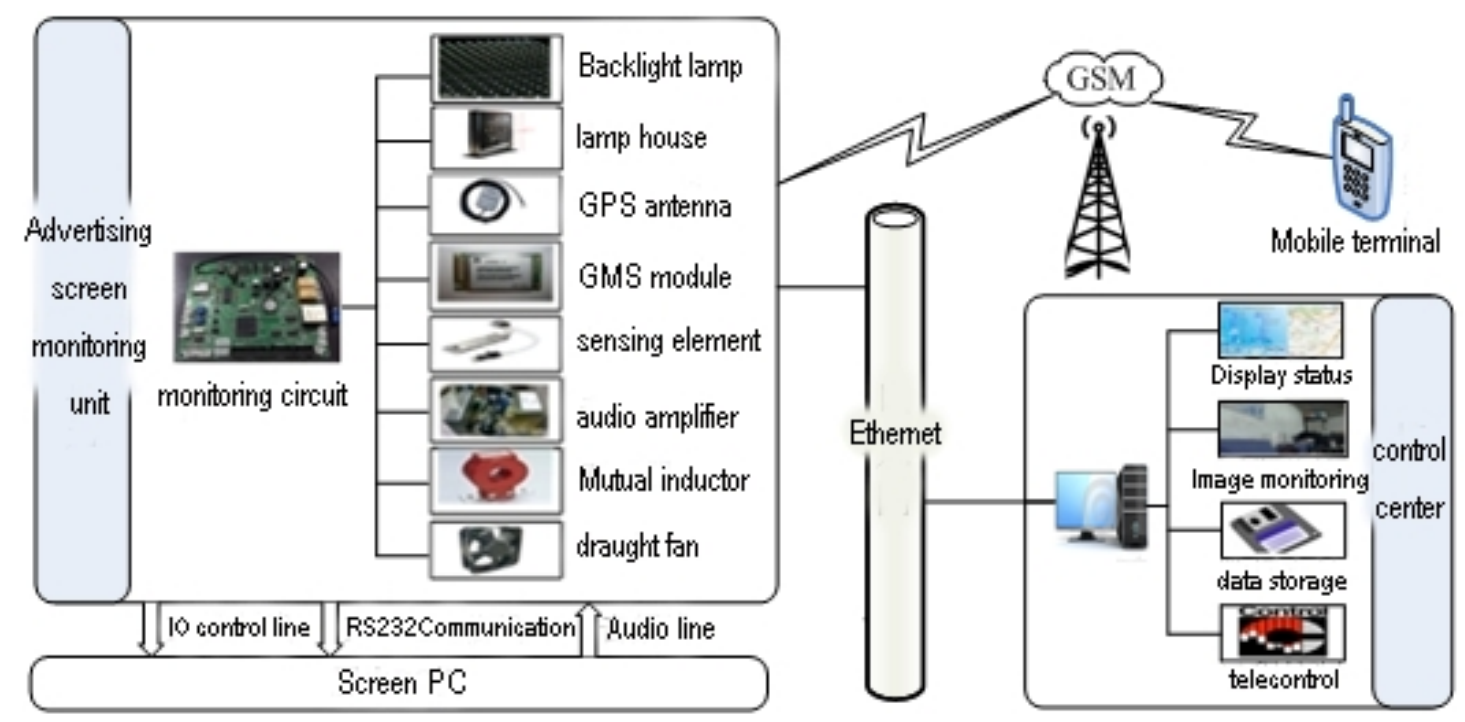

Fig. 1 System Structure Diagram

The control unit of the system is an embedded system which uses the Cortex-M3 kernel microcontroller as the core. Combined with the auxiliary circuit, the acquisition module, the control module, the storage module and the communication module, the system is specially designed for the remote monitoring and control of outdoor advertising screen. The design of the system structure of the monitoring unit of the advertisement screen is shown in Fig 2.2.

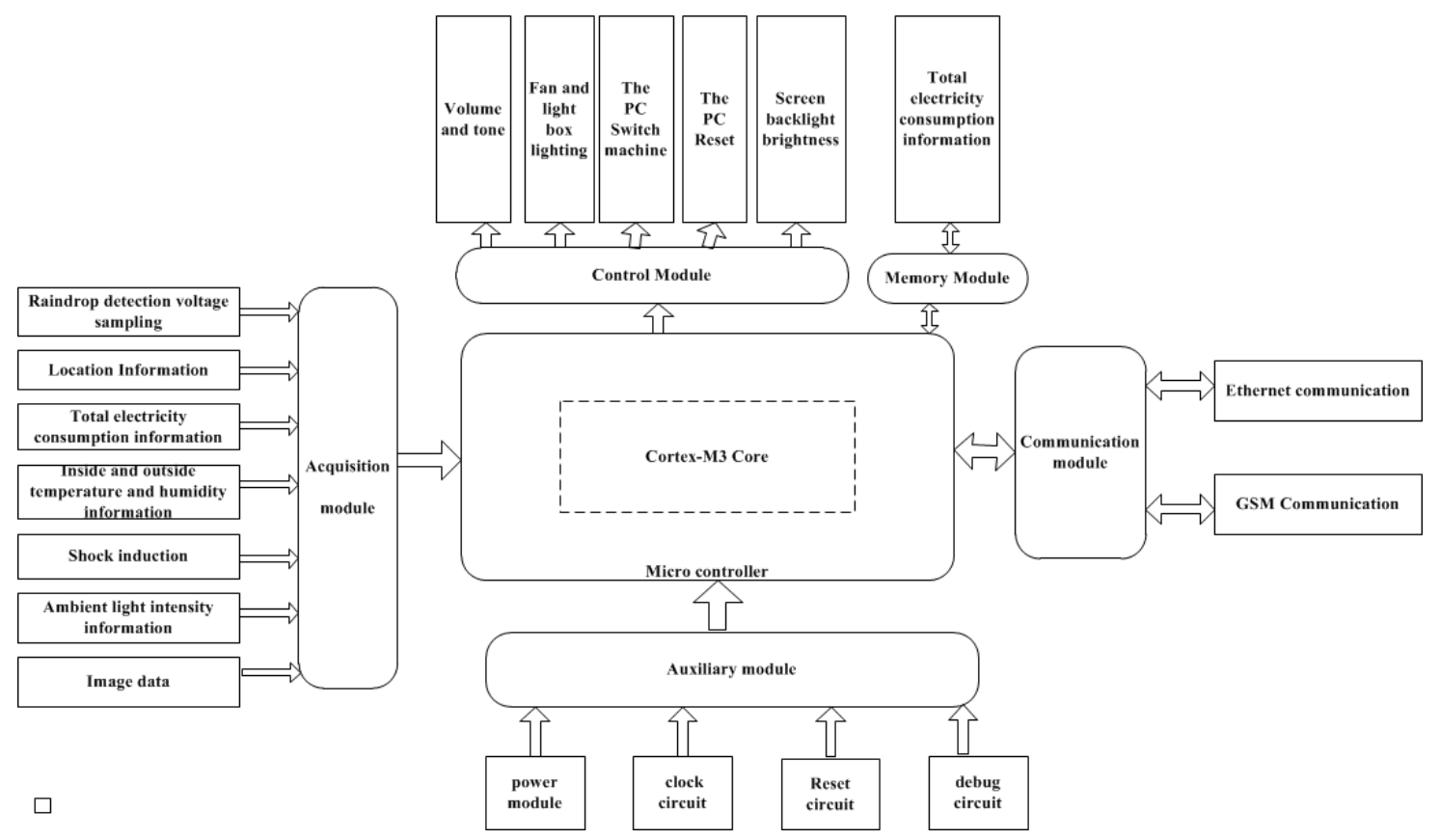

Figure.2 System Structure of the Advertisement Screen Monitoring Unit [4]

Based on the design idea of C/S model, the monitoring center uses the Visual Studio 2005 integrated development environment, combined with $\mathrm{C \#}$ programming language for design and development. The JavaScript scripting language is used in making maps. The monitoring center is mainly realized four major functions: state monitoring, image display, image storage and remote control. The function structure is shown in Fig 2.3. 


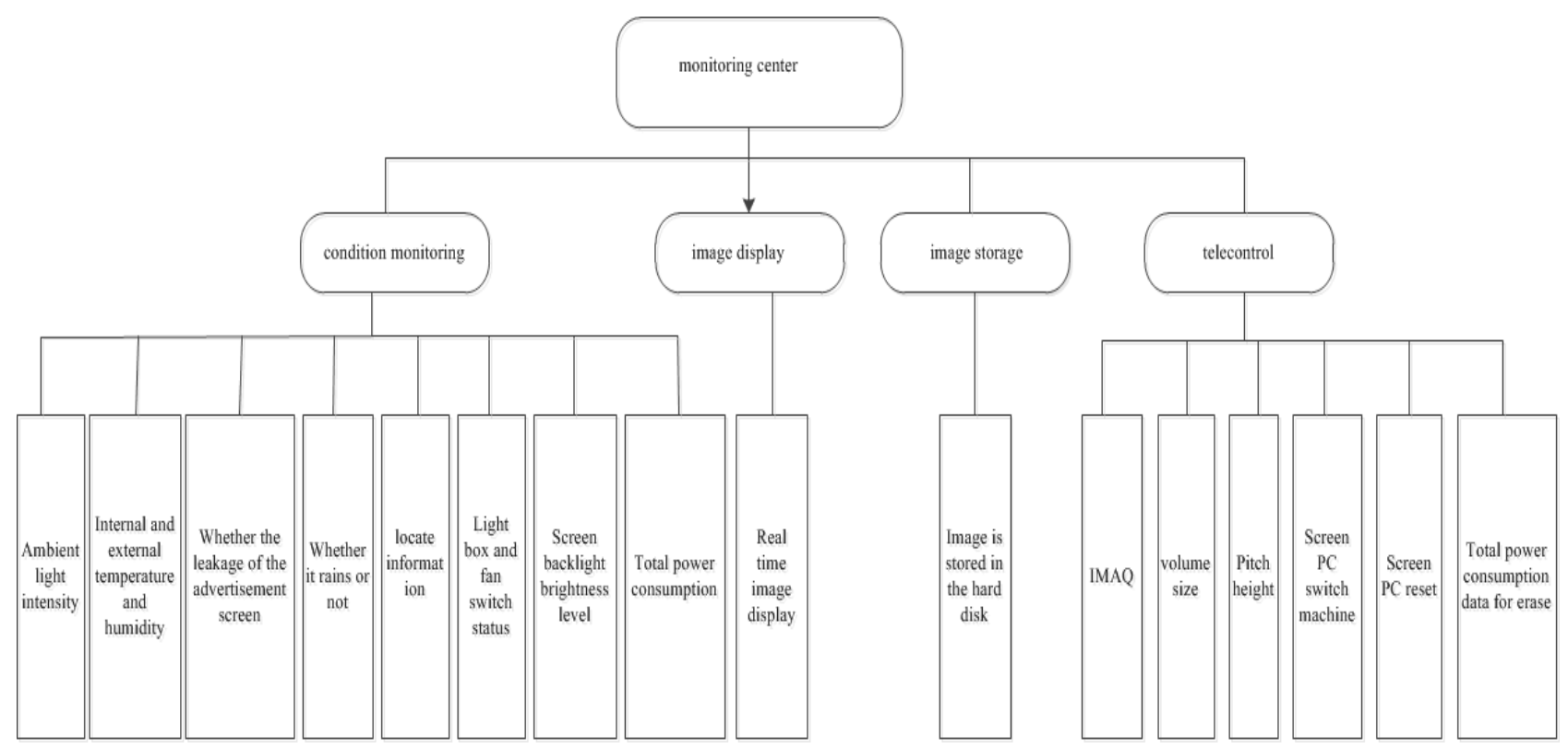

Figure. 3 Function Structure of Monitoring Center

\section{The Design of the remote monitoring unit of the advertisement screen}

A The hardware design of the remote monitoring unit of the advertisement screen

The hardware design of the remote monitoring unit is shown in Fig 3.1. The hardware part of the control unit is composed of STM32F103ZET6 acquisition module, control module, memory module, communication module and the corresponding auxiliary circuit. The micro controller is responsible for operating and processing data. The clock circuit provides a time base for the smallest system. Reset circuit is used to reset the minimum system. The serial port circuit is used to output the debugging procedure and the fast downloading procedure.

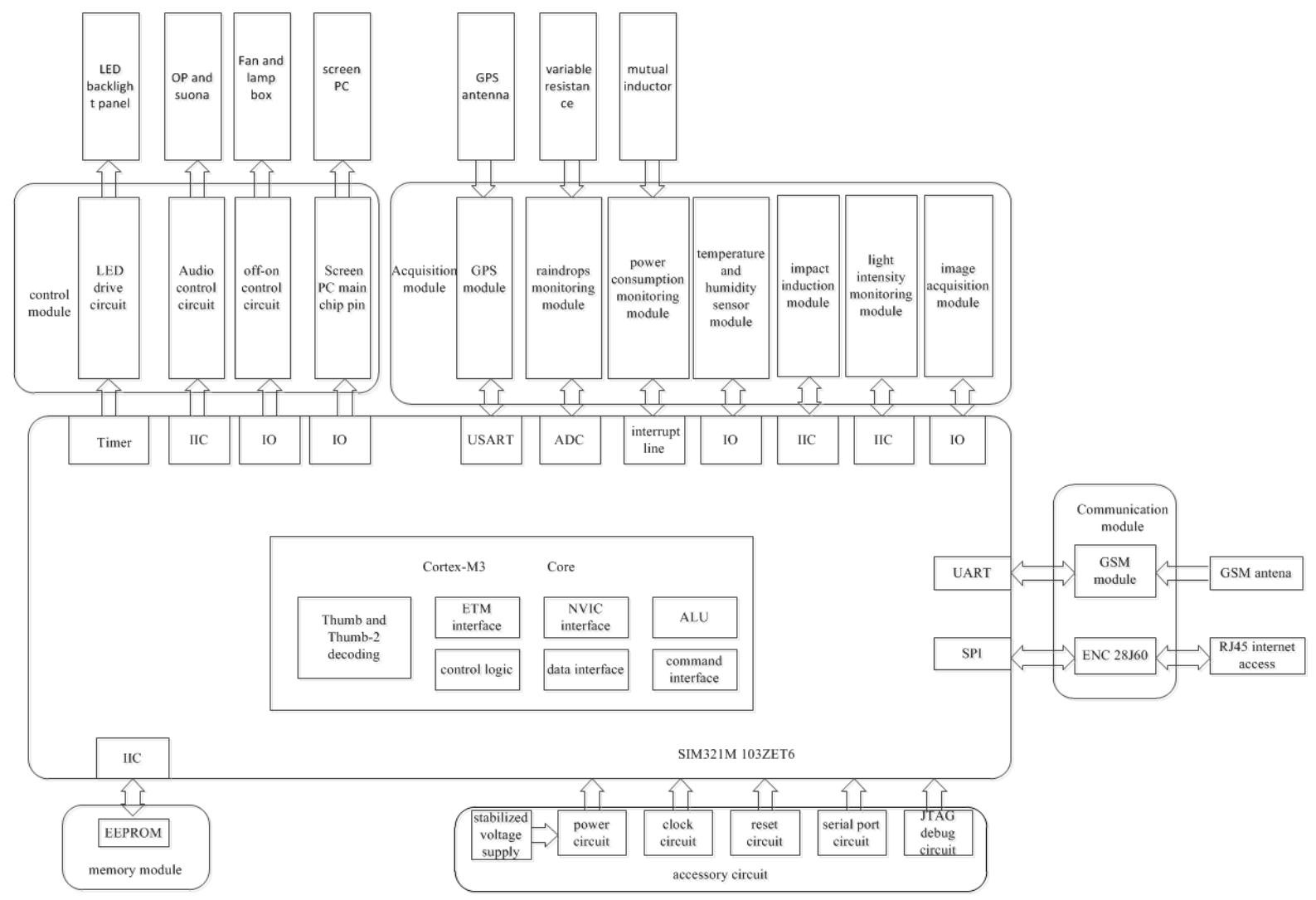

Figure.4 Structure of the Hardware of the Advertisement Screen Monitoring Unit [5] 
B The software design of the remote monitoring system of the advertisement screen

According to the embedded software design process, the software of the monitoring unit of the advertisement screen is designed in the bottom layer, the operating system layer and the application layer. Each task of software design has experienced the implementation of the underlying timing and drive, operating system management and scheduling, application implementation. The software system architecture is shown in Fig 3.2.

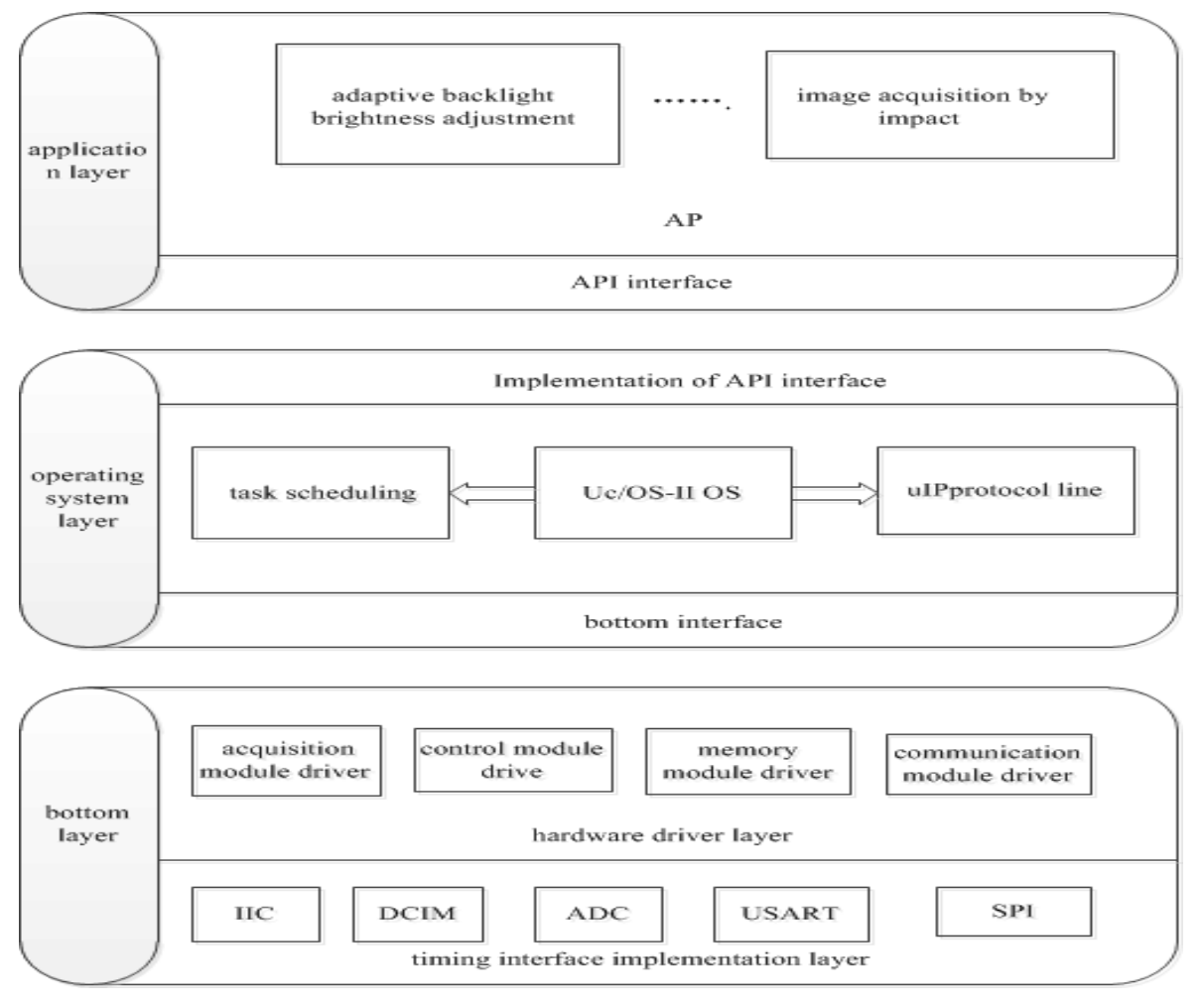

Figure.5 Software System Architecture

In the design of the underlying software, the hardware driver layer software needs the underlying timing's support, so the bottom layer of the underlying software is to achieve timing control. After the completion of timing control, the underlying driver of each module needs to be designed.

The operating system layer not only provides the UC/OS-II core for the system, but also implements the task scheduling. It packages the underlying driver, and provides the API function interface to the application layer calls. Although the UIP protocol stack does not need the support of the operating system, the network communication in real time will be better in combination with the operating system [6]. The sending and receiving function of UIP protocol stack calls the sending and receiving function of the underlying based on network chip to achieve data transceiver. UIP protocol stack also provides a function interface for the upper layer services.

The application program is developed and designed by calling the API interface functions provided by the operating system. The application program properly realizes the function of the whole system.

\section{The Software design of monitoring center based on C\#}

The system's advertising monitoring unit and monitoring center use the Ethernet as the communication mode. According to the 7 layers reference model of OSI, the Ethernet in the transport layer has the UDP and the TCP protocols. If the image data packet is lost during transmitting, the image displayed and saved in the monitoring center is incomplete, and the quality of the image does not meet the requirement of the system. The TCP has many advantages such as 
connection oriented and reliable. Besides, it has the timeout retransmission mechanism to ensure the reliability of data transmission. However fast the UDP transmits, its process is not reliable and is easy to lose packet. So this system applies the TCP [7].

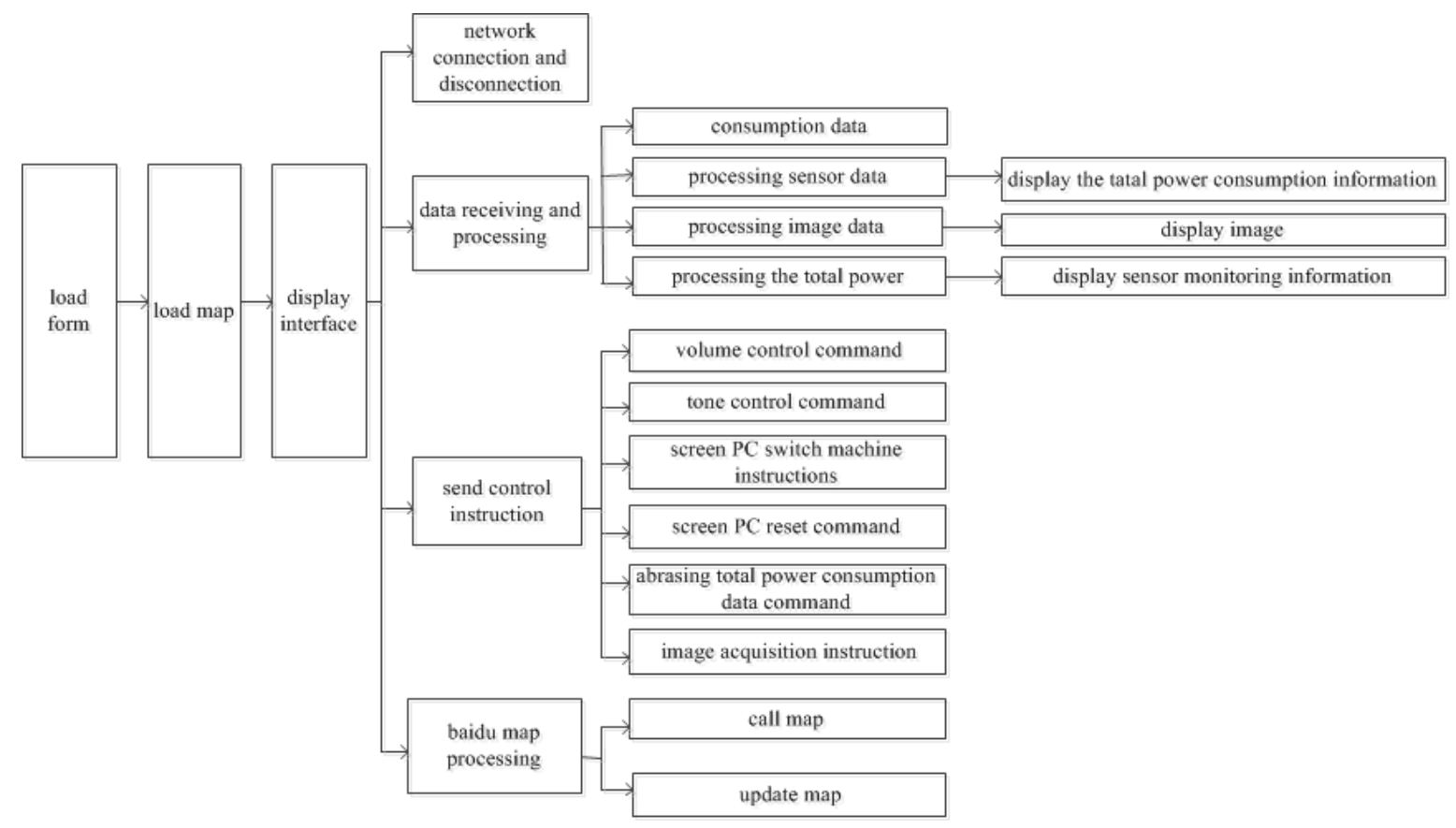

Figure.6 Software Design Structure of Monitoring Center [8]

The advertising screen monitoring unit uploads all the monitoring information to monitoring center through the network according to the custom protocol. The monitoring center not only can display and save the monitoring information, but also send the control commands to the advertisement screen for remote control. After completing the user interface design, the monitoring center software's design is based on four aspects: network connection and disconnection, data receiving and processing, instruction transmission and map processing as is shown in Fig 4.1. After the network connection is established successfully, the monitoring center comes into the process of the data receiving and processing. Once the monitoring center receives data, the data of sensor, the data of total power consumption and the data of image are processed according to the different types. The transmission of the control command includes the volume tone control instruction, the PC switch machine and the reset command, the total power consumption data instruction, the image collection instruction and so on. The monitoring center also achieves the call and updating of the map which can visually display the specific location of the ad screen.

\section{Conclusions}

The experimental results show that the outdoor advertising screen monitoring technology in this paper has characters of advanced, practical and so on.

1. The system realizes the GPS positioning function of the advertisement screen, the positioning accuracy is high and the coordinate drift is small.

2. Managers can remotely control the volume and the tone of the advertising screen, the volume and tone have 16 levels of adjustment range;

3. The advertising screen can monitor internal and external temperature and humidity, temperature and humidity with a resolution of $0.1{ }^{\circ} \mathrm{C}$, temperature accuracy within $\pm 1^{\circ} \mathrm{C}$, humidity accuracy is about $\pm 2 \% \mathrm{RH}$;

4. If the internal temperature of the advertising is too high, it can automatically control the fan to cool; 
5. Managers can remotely control the screen's PC to switch and reset, and also it can be set to timed switch;

6. The advertising screen backlight brightness can be self-adjusted according to the environment light intensity, and it has 16 levels of adjustment range;

7. It also has achieved the function of controlling the ad screen remotely through the text message and the monitoring center updates the monitoring data for about $5 \mathrm{~s}$.

Each module of the system operates normally without interference for a long time and also has strong adaptability. The monitoring information of sensor module is accurate. The indicators and functions of the system all meet the requirements. The system has good reliability and can be expanded application.

\section{References}

[1] Fang H M, Shang J, Qi F X, Zhao T. Types, Characteristics and Economic Value of Dynamic Advertising, Economic Forum. 32-33 (2008), p. 18

[2] Shen J Y. Mobile Internet Advertising Monitoring System: China, Patent CN103678595A. (2014).

[3] Zhai Y, Cheng X. Design of Smart Home Remote Monitoring System Based on Embedded System Computing, Control and Industrial Engineering (CCIE), 2011 IEEE 2nd International Conference on IEEE. 41- 44 (2011).

[4] Gerbacia W E, Gerbacia B E. Controlled and Monitored Remote Advertising and Information Display System: US, Patent US20070069973A1. (2007).

[5] Li F, Xia Y. Realization of Long-range Screen monitoring system, Microcomputer Information. 221-222 (2007), p. 23.

[6] Zhang L, Zhou Y F, Zhang J Y, Xie X G, Wu H J. Design of a Data Acquisition System for Production Equipments Based on uIP Protocols, Journal of University of Jinan (Science and Technology). 65-70 (2014), p. 28.

[7] Buchanan W J. TCP/UDP, Handbook of Data Communications \& Networks. 999-1018 (2004), p. 30(4).

[8] Sai S U, Zhu Z J, Duan Z, et al. Application of TCP Network Communication Based on C\#, Computer Programming Skills \& Maintenance. 89-90 (2013), p. 18. 